\title{
Effect of the Combination of Training and ERRa Inhibition on Liver Metabolism by Modulation of PDK4 and LXR- $\alpha$ Expression in STZ-Induced Diabetic and Healthy Rats
}

\author{
Yaser Masoumi-Ardakani ${ }^{1}$ (D), Soheil Aminizadeh ${ }^{1}$ (D), Beydolah Shahouzehi 2,3*(D) \\ 1 Physiology Research Center, Institute of Basic and Clinical Physiology Sciences, Kerman University of Medical Sciences, \\ Kerman, Iran \\ 2 Student Research Committee, Kerman University of Medical Sciences, Kerman, Iran \\ 3 Cardiovascular Research Center, Institute of Basic and Clinical Physiology Sciences, Kerman University of Medical \\ Sciences, Kerman, Iran \\ * Correspondence: bshahouzehi@gmail.com; b.shahozehi@kmu.ac.ir;
}

Scopus Author ID 36470992900

Received: 1.04.2020; Revised: 20.05.2020; Accepted: 23.05.2020; Published: 1.06.2020

\begin{abstract}
Diabetes is a public health problem that affects life quality. Exercise training (ET) and controlled dietary habits improve metabolic diseases such as diabetes. The mechanisms by which exercise training ameliorate metabolic diseases are not fully clear. We designed the current study to evaluate the combination of ERR $\alpha$ suppression and ET effects on the expression of LXR- $\alpha$, PDK4, and PPAR $\alpha$ in healthy and STZ-induced diabetic rats. Fifty-six male Wistar rats were divided into 8 groups $(\mathrm{n}=7)$ as follows; control, diabetic control (a single dose of $45 \mathrm{mg} / \mathrm{kg}$ of STZ), ERR $\alpha$ inhibition group (received $0.48 \mathrm{mg} / \mathrm{kg}$ of XCT790), endurance training, diabetic rats which received XCT790, diabetic rats which performed endurance training, rats which received XCT790 and performed endurance training, and diabetic rats which received XCT790 and also performed endurance training. Expression of the target gene and protein was carried out on the liver tissue. Our results showed that ET significantly increased PDK4, PPAR $\alpha$, and ERR $\alpha$ expression. ERR $\alpha$ suppression significantly increased LXR- $\alpha$ and PDK4 expression in healthy rats compared to the healthy control group. In the diabetic group with ERR $\alpha$ suppression, LXR- $\alpha$ expression significantly upregulated. The combination of ET and ERR $\alpha$ suppression did not change LXR- $\alpha$ expression compared to healthy and diabetic groups (CTL/ERR), but the expression of PDK4, PPAR $\alpha$, and ERR $\alpha$ was significantly upregulated.
\end{abstract}

Keywords: Endurance training; ERR $\alpha$; PDK4; PPAR $\alpha$; LXR $\alpha$; Diabetes.

(C) 2020 by the authors. This article is an open-access article distributed under the terms and conditions of the Creative Commons Attribution (CC BY) license (https://creativecommons.org/licenses/by/4.0/).

\section{Introduction}

The changes in lifestyle, including exercise training and improved dietary habits, can attenuate metabolic diseases such as diabetes, obesity, and insulin resistance [1]. Diabetes is a metabolic disease characterized by insulin deficiency or resistance and elevated blood glucose levels. Diabetes worldwide prevalence is increasing, and this was estimated that it would affect about $5 \%$ of the total population by the year 2025 . Therefore, proper management should be considered out to improve and/or treat diabetes and its deleterious consequences [2, 3].

The system capacity to adjust fatty acid (FA) and glucose oxidation are defined as metabolic flexibility. There is a competition between glucose and FA for oxidation, and this competition occurs at PDC (Pyruvate Dehydrogenase Complex) level. PDC catalyzes pyruvate oxidation to acetyl Co-A. The evidence from previous researches has suggested that PDC is 
precisely regulated by 4 kinase isozymes that designated as Pyruvate dehydrogenase kinases (PDKs) 1-4, and 2 phosphatases, including pyruvate dehydrogenase phosphatase 1 and 2 (PDP) [4-6]. The PDC phosphorylation and inactivation conducted by PDKs that caused fuel switch, and the metabolism goes toward FAs beta-oxidation and spares glucose oxidation. On the other hand, PDPs reverse PDKs inhibitory effect on PDC [4-6].

Estrogen-Related Receptors (ERRs) are a group of orphan receptors and transcriptional regulators of fuel (glucose and FAs) oxidation in mitochondria. Two ERR isoforms are ERR $\alpha$ and ERR $\beta$, and their expression is tissue-specific [7-9]. ERR $\alpha$ expressed ubiquitously and expressed specifically in muscle, heart, brain, and liver [10]. ERR $\alpha$ regulates fatty acid oxidation (FAO), mitochondrial biogenesis and metabolism, and also reduces gluconeogenesis in the liver, modulates cell differentiation, and metabolic syndrome [11, 12]. ERR $\alpha$ deletion (knockout) impaired mitochondrial gene expression pattern, but the basal state was not affected [10]. ERR $\alpha$ knockout mice showed increased Pepck (Phosphoenolpyruvate carboxykinase; a gluconeogenic enzyme) activity in the liver that demonstrating ERR $\alpha$ suppressive role on gluconeogenesis. On the other hand, ERR $\alpha$ inhibition reduced metabolic pathways and oxidative gene expression in mitochondria [12]. ERR $\alpha$ is considered as a therapeutic target for diabetes treatment; it reduced hepatic glucose production and elevated muscle oxidative capacity [13].

Peroxisome proliferator-activated receptor (PPAR) family of transcription factors regulating glucose and lipid metabolism, inflammation, apoptosis, cell proliferation, and differentiation [14]. There are three isoforms of PPARs, including PPAR $\alpha, \operatorname{PPAR} \beta / \delta$, and PPAR $\gamma$. PPAR $\alpha$ and PPAR $\beta$ regulate FAO, but the latter is involved in the differentiation of adipocytes. PPAR $\alpha$ mainly expressed in tissues such as the liver, intestine, kidney, skeletal muscle, and heart and plays an important role in lipid metabolism and FAO, oxidative stress, and also inflammatory response modulation $[10,14]$. PPAR $\alpha$ is located in the center of energy balance, which makes PPAR $\alpha$ a vital receptor affecting disease development that is related to obesity, including diabetes, insulin resistance, and metabolic syndrome [10, 14].

Liver X Receptors (LXRs) are DNA-binding and ligand-activated transcription factors, previously considered as orphan nuclear receptors, that have been discovered that activated by oxysterols. LXRs are implicated in carbohydrate, cholesterol, and triacylglycerol (TG) metabolism. There are two members of LXR subfamily that have high similarity to each other (78\%) and designated as LXR- $\alpha$ and LXR- $\beta$ [15]. LXR- $\alpha$ expressed in tissues including; liver, kidney, adipose tissue, and adrenals, whereas LXR- $\beta$ is ubiquitously expressed. Multiple lines of evidence suggest that LXRs are involved in bile acids production, hepatic lipogenesis, and cholesterol homeostasis [15-18]. LXR- $\alpha$ causes reverse cholesterol transport (RCT) and lipogenesis in the liver by up-regulation of ATP-binding cassette A1 (ABCA1) and Sterol response element-binding protein-1c (SREBP-1c), respectively [15].

Endurance training (ET) is considered as a beneficial form of exercise that showed valuable properties including reduced body weight, attenuated plasma glucose concentrations, and ameliorated lipid profile and insulin resistance (IR) in diabetes [19-21]. The adaptations in skeletal muscle as a result of ET involve an increase in the capacity for aerobic metabolism made possible by an adaptive increase in mitochondrial content as well as a number of other enzymatic adaptations that may contribute to the altered metabolic response to exercise in the trained state [22].

Skovgaard et al. (2016) showed that PDK4 mRNA increases with endurance exercise training [23]. It has been reported that prolonged low-intensity and short-term high-intensity 
exercise lead to remarkable PDK4 gene expression. Eight weeks of exercise upregulated PDK4 expression and inhibited PDC activity and, therefore, glucose oxidation [6]. It was reported that ET increased PDK4 protein expression and activity and finally decreased lipogenesis [5]. Exercise training and ERR $\alpha$ regulate PPAR $\alpha$ expression [24]. Tunstall et al. did not find a significant change of PPAR $\alpha$ expression after ET [25]. But, Horowitz and colleagues reported that ET significantly increased PPAR $\alpha$ expression [26]. PPAR $\alpha$ activation induces PDK4 upregulation [27]. Also, this was showed that exercise training affects LXR- $\alpha$ and PPAR $\alpha$ expression $[21,26,28,29]$. PPAR $\alpha$ increased FAO, and LXR- $\alpha$ promotes lipogenesis and downregulates FAO. Also, PDK4 activation favors FAO and reduces glucose oxidation [14]. on the other hand, it has been well documented that disturbed FAO in mitochondria and elevated Nonesterified fatty acid (NEFA) uptake increase pro-inflammatory lipids (fatty acyl-CoA and ceramide) and TG accumulation that inactivate insulin signaling which finally results in insulin resistance [30]. Therefore, PPAR $\alpha$ and PDK4 dysregulations lead to defects in FAO oxidation, which finally disturbed insulin signaling and deteriorate diabetes state.

The mechanisms by which exercise training ameliorate metabolic diseases are not fully clear. On the other hand, ET enhanced glucose and FA utilization, and understanding the mechanisms mediating these effects may be important for the treatment of type 2 diabetes. ERR $\alpha$ has also attracted attention as a potentially important intervention for diabetes management. In the current study, we aimed to evaluate the combination of ERR $\alpha$ inhibition and ET effects on the expression of LXR- $\alpha$ and PDK4 in healthy and STZ-induced diabetic rats.

\section{Materials and Methods}

\subsection{Materials.}

XCT790 (Sigma, No: X4753), STZ (Sigma, No: S0130), Total RNA isolation kit (BioBasic; BS414), cDNA synthesis kit (TAKARA; RR037A), SYBR Green (Ampliqon; A325402), and the antibodies which used in this study were including LXR $\alpha / \beta$ (sc-271064) and $\beta$-actin (sc-47778) from Santacruz biotechnologies and PDK4 antibody (PAB5916) obtained from Abnova Corporation. Polyvinylidene Difluoride membrane (Amersham Hybond P 0.45 PVDF, 15289894) and Enhanced Chemiluminescence substrate (ECL Plus Western Blotting Substrate, 32106) were obtained from GE Healthcare and Thermo Scientific, respectively.

\subsection{Ethical Approval.}

All animal procedures were conducted in accordance with the requirements of the Declaration of Helsinki (http://www.wma.net/e/policy/pdf/17c.pdf). Also, this study was approved by the ethics committee of Kerman Medical University (IR.KMU.REC.1394.633).

\subsection{Methods.}

\subsubsection{ERR $\alpha$ disruption.}

ERR $\alpha$ suppression was performed by daily XCT790 $(0.48 \mathrm{mg} / \mathrm{kg})$ intraperitoneal injections for 28 days [11, 31]. 


\subsubsection{Diabetes induction.}

We used a single intraperitoneal injection of STZ $(45 \mathrm{mg} / \mathrm{kg}$ prepared in $0.1 \mathrm{M}$ citrate buffer, $\mathrm{pH} 4.5$ ) for diabetes induction in this study. In order to confirm diabetes in animals, 72 $\mathrm{h}$ after STZ injection in overnight fasted animals, the blood glucose was quantified by glucometer (Accu-Check, Germany) and rats with FBG (Fasting Blood Glucose) higher than $250 \mathrm{mg} / \mathrm{dl}$ were considered as diabetic and entered to the study.

\subsubsection{Endurance training (ET) protocol.}

The animals in this study performed ET for 4 weeks ( 5 days per week). The trained groups were familiarized with treadmill running protocol at low speeds $(15-20 \mathrm{~m} / \mathrm{min})$ for 20 $\mathrm{min} /$ day for the first 5 days of the study. Then, the duration increased gradually over the 4 week period, and the animals were running for $50 \mathrm{~min} /$ day at $27 \mathrm{~m} / \mathrm{min}$ in the last 2 weeks [32].

\subsubsection{Animal study.}

Fifty-six male Wistar rats were used in the present study. The animals were maintained at a controlled condition, light/dark cycle, $24 \pm 2^{\circ} \mathrm{C}$, and free access to food and water. There was an acclimatization period for a week, and after that, the animals were divided into 8 groups $(n=7)$ as follows; Control untreated group that received no treatments (group 1), Diabetic control group that received a single dose of $45 \mathrm{mg} / \mathrm{kg}$ of STZ (group 2), ERR $\alpha$ disrupted group that received $0.48 \mathrm{mg} / \mathrm{kg}$ of XCT790 (group 3), ET group (group 4), diabetic rats which received XCT790 (group 5), diabetic rats that performed ET (group 6), rats that received XCT790 and performed ET (group 7) and diabetic rats that received XCT790 and also performed ET (group 8). At the end of the study, the animals were sacrificed, and liver tissue was dissected and washed with cold saline. Finally, the liver was freeze by liquid nitrogen and stored at $-80^{\circ} \mathrm{C}$ until real-time PCR and western blotting measurements.

\subsubsection{RNA extraction and real-time PCR.}

Liver tissue $(50 \mathrm{mg}$ ) was dissected and homogenized at lysis buffer by sonicator (Heilscher H200, Germany). Total RNA was extracted according to the kit protocol. Then, complementary DNA (cDNA) was synthesized from extracted RNA (500 ng) by cDNA synthesis kit according to its procedure. Real-time PCR was performed by a specific primer (Table 1). The real-time PCR reaction was contained $10 \mu \mathrm{l} \mathrm{SYBR}$ green, $1 \mu \mathrm{l}$ of forwarding and $1 \mu \mathrm{l}$ of Reverse primers, $100 \mathrm{ng}$ of synthesized cDNA, and finally, the reaction volume reached to $20 \mu \mathrm{l}$ by $\mathrm{dH}_{2} \mathrm{O}$. The annealing temperatures were according to each primer's Tm. Generally, the thermal protocol was as follows; $95^{\circ} \mathrm{C}(5 \mathrm{~min}), 95^{\circ} \mathrm{C}(15 \mathrm{sec})$, annealing temperature (45 sec), 40 cycles, and after cycles were done, the melt curve analysis was performed. We used 18S rRNA as a housekeeping gene. The relative expression of genes was determined by ${ }^{2-\Delta \Delta \mathrm{Ct}}$ method [33].

Table 1. Primers sequence which used in for real-time PCR gene expression measurement.

\begin{tabular}{l|l|l|l}
\multicolumn{2}{|c}{ Gene } & \multicolumn{1}{c}{ Forward Sequence } & Reverse Sequence \\
$\mathbf{1}$ & LXR $\alpha$ & CCTGATGTTTCTCCTGACTC & TGACTCCAACCCTATCCTTA \\
\hline $\mathbf{2}$ & ERR $\alpha$ & AAGCCCTGATGGACACCTC & GAAGCCTGGGATGCTCTTG \\
\hline $\mathbf{3}$ & PDK4 4 & AAGCCCTGATGGACACCTC & GAAGCCTGGGATGCTCTTG \\
\hline $\mathbf{4}$ & PPAR $\alpha$ & GATACCACTATGGAGTCCACGCA & GCCGAAAGAAGCCCTTGC \\
\hline $\mathbf{5}$ & $18 \mathrm{~S}$ & GCAATTATTCCCCATGAACG & GGCCTCACTAAACCATCCAA \\
\hline
\end{tabular}




\subsubsection{Western Blotting.}

In order to liver tissue homogenization and protein extraction, we utilized RIPA buffer (Contained Protease Inhibitor, Phenylmethylsulfonyl Fluoride, and Sodium Orthovanadate [1 $\mathrm{mM}$ of each one], $\mathrm{pH}$ 7.4). Forty $\mathrm{mg}$ of each liver sample was obtained and homogenized on ice-cold RIPA buffer by Ultrasonic Processor (Hielscher, UP200H, Germany). The obtained homogenate was centrifuged (At $4{ }^{\circ} \mathrm{C}, 20000 \mathrm{rpm}$, for $20 \mathrm{~min}$ ), and then the supernatants were collected for further study. Bradford method was performed to quantify the protein concentration in obtained supernatant. An equal volume of each sample supernatant and $2 \mathrm{X}$ sample buffer incubated at $95^{\circ} \mathrm{C}$ for 5 minutes and then 80 micrograms of protein was loaded into wells, and SDS-PAGE (sodium dodecyl sulfate-polyacrylamide gel electrophoresis) on a $12.5 \%$ gel was conducted under controlled condition (120 V, $80 \mathrm{~min}$, Tris-Glycine running buffer $\mathrm{pH}$ 8.3). After protein separation in gel, proteins were transferred to a PVDF membrane (P 0.45 PVDF) that was activated by methanol. Transfer performed at a constant current 220 $\mathrm{mA}$ in cold transfer buffer for $90 \mathrm{~min}$. Then after, the PVDF membrane was incubated with 5 $\%$ skim milk in tris-buffered saline and Tween 20 (TBST) as blocking buffer on a shaker for 2 hours, and then it washed 4 times with TBST (for 5 min each time). Incubation with primary antibody was 1 hour, and after washing (4 times each $5 \mathrm{~min}$ ), final incubation with secondary antibody 1 hour followed by 4 times washing was performed. The membrane was incubated with Enhanced Chemiluminescence substrate (ECL Plus Substrate) for about 90 seconds and an antigen-antibody complex detected by ECL detection film in a dark room. The ImageJ software was used to analyze the protein bands densities; also, the $\beta$-Actin protein was used as housekeeping control [33].

\subsection{Statistical Analysis.}

The data were analyzed by two-way analysis of variance (Two-Way ANOVA) test, and Tukey's method was used to pairwise comparisons of the studied groups. The analysis in this study was performed by SPSS (version 22) and Sigma Plot (version 12) soft wares. Data are expressed as Mean \pm SEM. The $p$ values $<0.05$ were considered significant.

\section{Results and Discussion}

Our results showed that ET significantly reduced LXR- $\alpha$ gene expression in the diabetic group compared to diabetic control rats $(\mathrm{p}=0.002)$ (Figure 1). On the other hand, ET increased PDK4, PPAR $\alpha(p<0.001)$, and ERR $\alpha(p=0.031)$ expression in healthy and diabetic rats compared to their control counterparts (CTL), significantly (Figures. 2-4).

ERR $\alpha$ suppression $(C T L / E R R)$ significantly increased LXR- $\alpha(p<0.001)$ and PDK4 $(\mathrm{p}=0.011)$ expression in healthy rats compared to the healthy control group (CTL) (Figures. 1 and 2). In the diabetic group with ERR $\alpha$ suppression, the LXR- $\alpha$ expression significantly upregulated ( $\mathrm{p}<0.001)$ compared to the diabetic control group (CTL) (Figure 1).

The combination of ET and ERR $\alpha$ suppression (ET/ERR) did not change LXR- $\alpha$ expression compared to healthy and diabetic groups (CTL/ERR), but the expression of PDK4, PPAR $\alpha$ and ERR $\alpha$ were significantly upregulated (Figures. 2-4).

The combination of ET and ERR $\alpha$ suppression (ET/ERR) in the healthy group increased PDK4 and PPAR $\alpha$ expression $(\mathrm{p}<0.001)$ compared to the healthy group with ERR $\alpha$ inhibition (Health-CTL/ERR) (Figures. 2 and 3). 


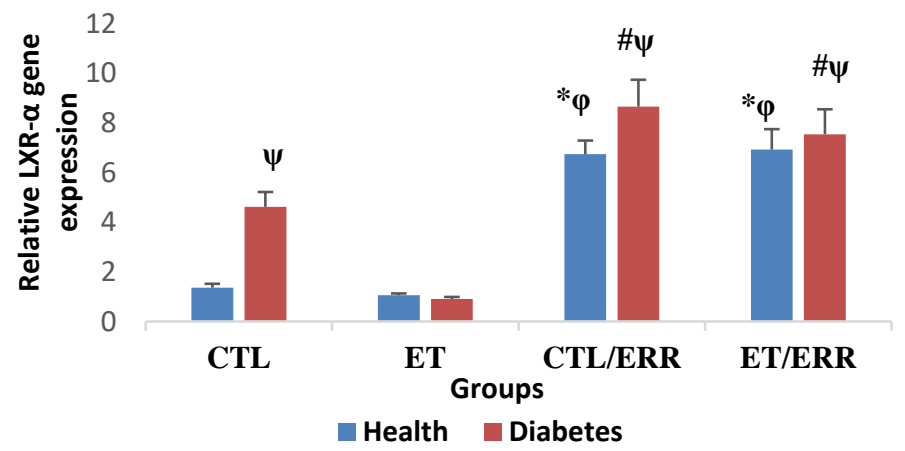

Figure 1. Relative LXR- $\alpha$ gene expression quantified by Real-Time PCR method in liver of studied groups. The groups were as follows; Untreated Control (CTL); Endurance training (ET); Health/Diabetic Control animals which ERR $\alpha$ expression was inhibited by XCT (CTL/ERR); Health/Diabetic animals which ERR $\alpha$ was inhibited by XCT and performed ET (ET/ERR). * statistically significant to health control group, \# statistically significant to diabetic control group, $\varphi$ statistically significant to ET in healthy rats, $\psi$ statistically significant to ET in diabetic rats. Data are expressed as Mean \pm SEM. ( $<<0.05$ was considered as significant).

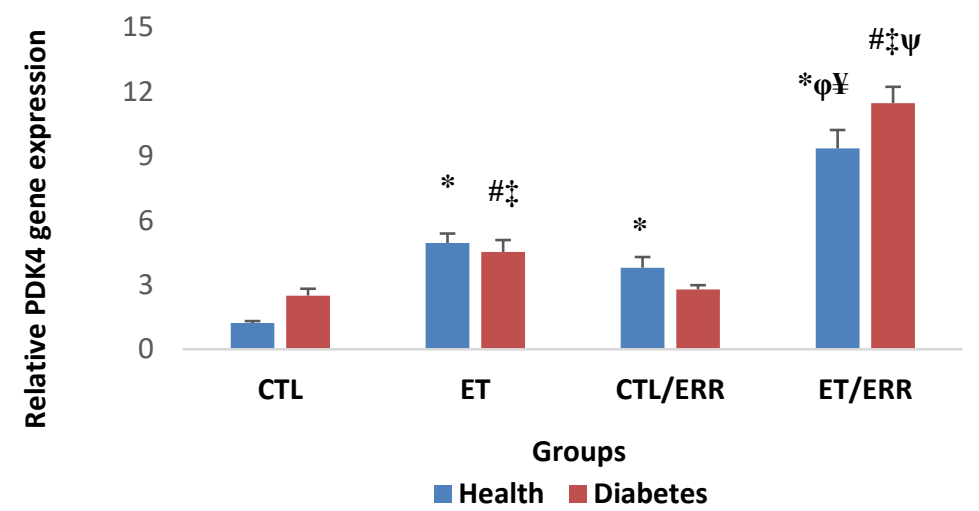

Figure 2. Relative PDK4 gene expression quantified by Real-Time PCR method in liver of studied groups. The groups were as follows; Untreated Control (CTL); Endurance training (ET); Health/Diabetic Control animals which ERR $\alpha$ expression was inhibited by XCT (CTL/ERR); Health/Diabetic animals which ERR $\alpha$ was inhibited by XCT and performed ET (ET/ERR). * statistically significant to health control group, \# statistically significant to diabetic control group, $\varphi$ statistically significant to ET in healthy rats, $¥$ statistically significant to health CTL/ERR (Healthy animals which ERR $\alpha$ was inhibited), $\psi$ statistically significant to ET in diabetic rats, $\$$ statistically significant to diabetic CTL/ERR (Diabetic animals which ERR $\alpha$ was inhibited). Data are expressed as Mean \pm SEM. ( $p<0.05$ was considered as significant).

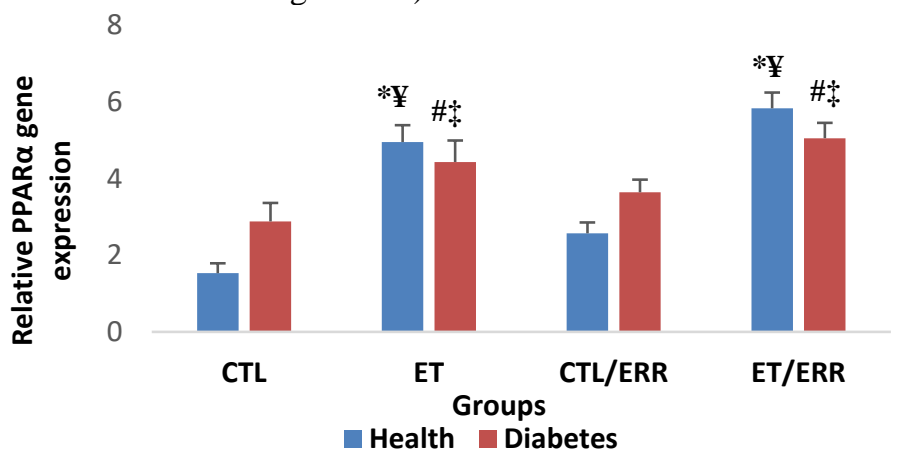

Figure 3. Relative PPAR $\alpha$ gene expression quantified by Real-Time PCR method in liver of studied groups. The groups were as follows; Untreated Control (CTL); Endurance training (ET); Health/Diabetic Control animals which ERR $\alpha$ expression was inhibited by XCT (CTL/ERR); Health/Diabetic animals which ERR $\alpha$ was inhibited by XCT and performed ET (ET/ERR). * statistically significant to health control group, \# statistically significant to diabetic control group, $\varphi$ statistically significant to ET in healthy rats, $¥$ statistically significant to health CTL/ERR (Healthy animals which ERR $\alpha$ was inhibited), $\psi$ statistically significant to ET in diabetic rats, \$ statistically significant to diabetic CTL/ERR (Diabetic animals which ERR $\alpha$ was inhibited). Data are expressed as Mean \pm SEM. ( $p<0.05$ was considered as significant). 


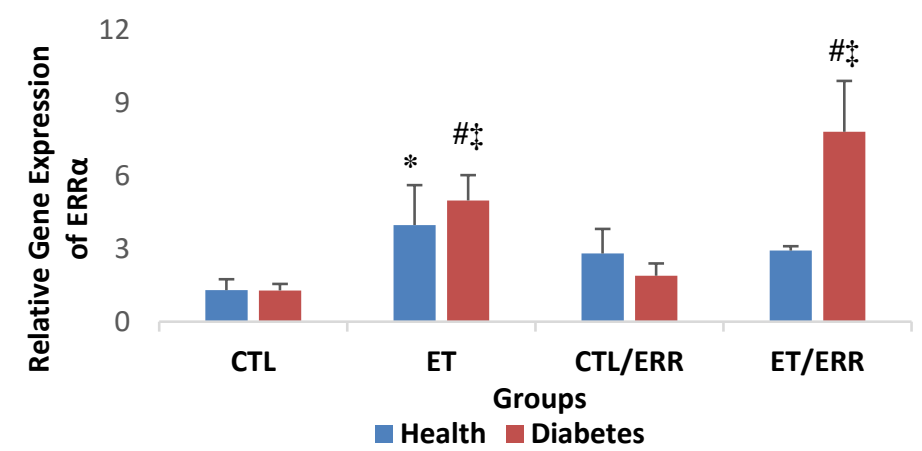

Figure 4. Relative ERR $\alpha$ gene expression quantified by Real-Time PCR method in liver of studied groups. The groups were as follows; Untreated Control (CTL); Endurance training (ET); Health/Diabetic Control animals which ERR $\alpha$ expression was inhibited by XCT (CTL/ERR); Health/Diabetic animals which ERR $\alpha$ was inhibited by XCT and performed ET (ET/ERR). * statistically significant to health control group, \# statistically significant to diabetic control group, $\$$ statistically significant to diabetic CTL/ERR (Diabetic animals which ERR $\alpha$ was inhibited). Data are expressed as Mean \pm SEM. ( $<<0.05$ was considered as significant).

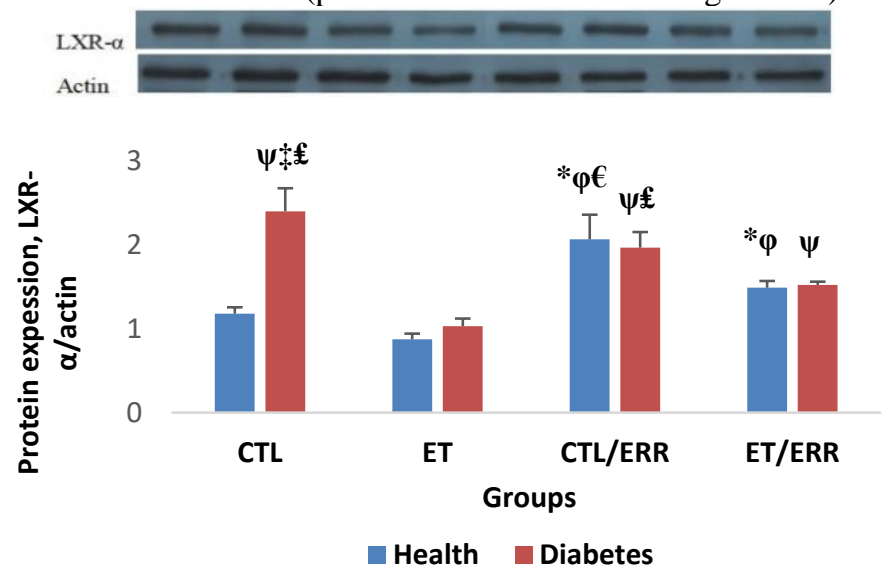

Figure 5. LXR- $\alpha$ protein expression measured by western blotting in liver of the studied groups; The groups were as follows; Untreated Control (CTL); Endurance training (ET); Health/Diabetic Control animals which ERR $\alpha$ expression was inhibited by XCT790 (CTL/ERR); Health/Diabetic animals which ERR $\alpha$ was inhibited by XCT790 and performed ET (ET/ERR). * statistically significant to health control group, $\varphi$ statistically significant to ET in healthy rats, $\psi$ statistically significant to ET in diabetic rats, $\ddagger$ statistically significant to diabetic CTL/ERR (Diabetic animals which ERR $\alpha$ was inhibited), $€$ statistically significant to healthy rat with ET and $\mathrm{ERR} \alpha$ suppression (ET/ERR), £ statistically significant to diabetic rat with ET and ERR $\alpha$ suppression (ET/ERR). Data are expressed as Mean \pm SEM. ( $\mathrm{p}<0.05$ was considered as significant).

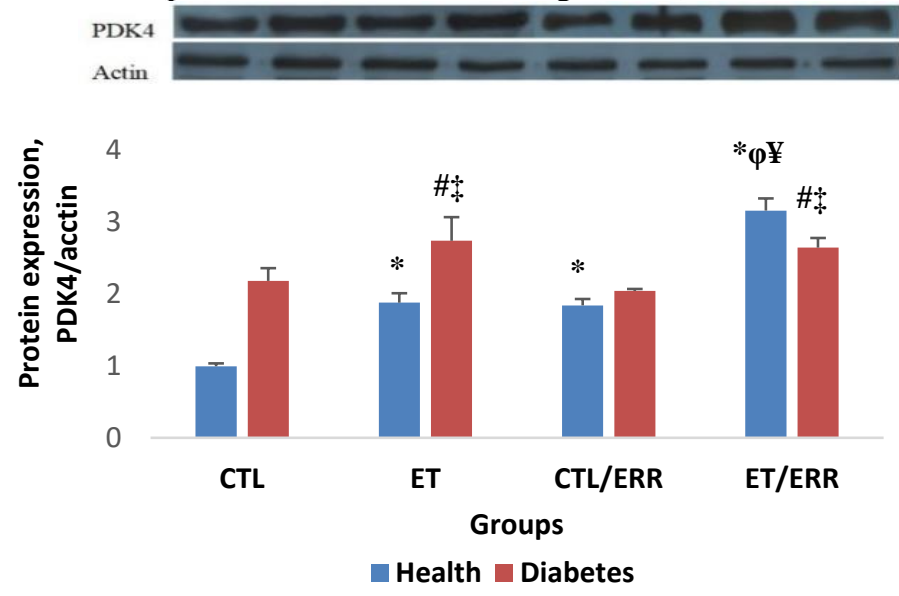

Figure 6. PDK4 protein expression measured by western blotting in liver of the studied groups; The groups were as follows; Untreated Control (CTL); Endurance training (ET); Health/Diabetic Control animals which ERR $\alpha$ expression was inhibited by XCT790 (CTL/ERR); Health/Diabetic animals which ERR $\alpha$ was inhibited by XCT790 and performed ET (ET/ERR). * statistically significant to health control group, \# statistically significant to diabetic control group, ¥ statistically significant to health CTL/ERR (Healthy animals which ERR $\alpha$ was inhibited), $\varphi$ statistically significant to ET in healthy rats, $\psi$ statistically significant to ET in diabetic rats, $\ddagger$ statistically significant to diabetic CTL/ERR (Diabetic animals which ERR $\alpha$ was inhibited). Data are expressed as Mean \pm SEM. ( $p<0.05$ was considered as significant). 
Also, the combination of ET and ERR $\alpha$ suppression (ET/ERR) in the diabetic group significantly increased PDK4, ERR $\alpha(p<0.001)$ and PPAR $\alpha(p=0.021)$ expression compared to the diabetic group with ERR $\alpha$ inhibition (Diabetic-CTL/ERR).

LXR- $\alpha$ protein expression significantly increased in STZ-induced diabetic rats (CTL) compared to the other 3 groups with diabetes. The combination of ET and ERR $\alpha$ suppression (ET/ERR) did not significantly change LXR- $\alpha$ protein expression compared to (CTL/ERR0 in healthy and diabetic groups (Figure 5). In STZ-induced diabetic rats, the PDK4 protein expression was increased (Figure 6). ERR $\alpha$ inhibition (CTL/ERR) increased PDK4 protein expression compared to the healthy control group (CTL) ( $\mathrm{p}<0.001)$. The combination of ET and ERR $\alpha$ suppression (ET/ERR) in the healthy group increased PDK4 protein expression compared to healthy control (CTL), ET, and health CTL/ERR groups ( $\mathrm{p}<0.001)$. In diabetic rats, ET and a combination of ET and ERR $\alpha$ suppression (ET/ERR) significantly increased PDK4 protein expression compared to the diabetic CTL/ERR group ( $\mathrm{p}<0.001$ ) (Figure 6).

In the present study, we examined ET and ERR $\alpha$ inhibition effects, alone and in combination with LXR- $\alpha$, PDK4, and PPAR $\alpha$ expression in the liver of healthy and diabetic rats. The beneficial effects of exercise training and especially ET on carbohydrate and lipid metabolism, were reported by several studies [21,34,35]. ET maintains carbohydrate and lipid homeostasis in such a way that ET increase FAO, spare glucose oxidation, improves insulin sensitivity and considered a promising intervention to manage diabetes [21,34,35].

The PDC reversible phosphorylation by PDKs results in PDC inactivation and directing the metabolism toward FAO [4,6]. There is a body of evidence that reported that PDK4 expression was upregulated in diabetes either in human or animal models [4-6,30]. Our finding over PDK4 expression that was upregulated in STZ-induced diabetic rats in this study is in accordance with previous studies [4,6,30]. There are several reports about exercise training effects on PDK4 expression [4-6]. ET in this study increased PDK4 expression in healthy and diabetic groups at gene and protein levels. Our results confirmed previous studies that reported that training regulates PDK4 expression. Shortly, it has been reported that ET and highintensity exercise increased PDK4 expression in skeletal muscle [5]. Also, it was reported that eight weeks of exercise increased PDK4 expression and therefore decreased PDC activity and glucose oxidation [6]. Abreu and coworkers (2016) reported that ET increased PDK4 protein and reduced ACC activity and, as a result, decreased lipogenesis [4].

It has been reported that ERR $\alpha$ promoted PDK4 gene transcription and therefore increased PDK4 levels [4,36]. We found that ERR $\alpha$ suppression reduced PDK4 expression in diabetic rats. Hence, these findings are along with ERR $\alpha$, promoting effects on PDK4 expression [36]. But, the combination of ET with ERR $\alpha$ inhibition remarkably increased PDK4 expression in healthy and diabetic rats. These findings indicated that ET effect on PDK4 expression is probably independent of ERR $\alpha$, where ERR $\alpha$ was neutralized, ET increased PDK4 gene, and protein expression. The possible mechanism behind this finding may be related to ERR $\gamma$. It has been reported that when ERR $\alpha$ is knockout, ERR $\gamma$ showed a compensatory response and take same responsibility as ERR $\alpha$. But this thesis in this study needs further examination and should be proved, especially; when we did not observe this possible compensatory effect in a group that only ERR $\alpha$ was inhibited. In diabetes, PDK4 expression and activity will be upregulated, which results in decreased glucose oxidation in the liver [4]. PDK4 up-regulation limits oxidation of glucose and activates FAO, which probably helps to decrease NEFA in serum and improve insulin signaling in diabetes, which confirms the beneficial effects of ET and ERR $\alpha$ inhibition together. 
Our findings indicated that ET in diabetic rats significantly reduced LXR- $\alpha$ gene and protein expression compared to the diabetic control group. These findings are along with exercise training effects where running increase FAO and reduce lipogenesis [35]. LXR- $\alpha$ is involved in lipid synthesis by affecting SREBP-1c and cholesterol homeostasis by targeting ABCA1 [15,16,18]. Therefore, ET improves lipid homeostasis in STZ-induced diabetic rats. ERR $\alpha$ improves mitochondria oxidative pathway, induce FAO, and mitochondrial biogenesis $[13,36]$.

It has been shown that ERR $\alpha$ knockout in mice results in reduced serum glucose levels and improved insulin sensitivity. Our data showed that ERR $\alpha$ inhibition (CTL/ERR) in healthy and diabetic groups increased LXR- $\alpha$ gene and protein expression and interestingly, ET did not reduce LXR- $\alpha$ expression in groups with a combination of ET and ERR $\alpha$ suppression (ET/ERR - healthy and diabetic group). It seems that ET reducing effects on LXR- $\alpha$ expression is dependent on ERR $\alpha$; therefore, in the group with a combination of ET and ERR $\alpha$ inhibition we did not observe ET lowering effects on LXR- $\alpha$ expression compared to groups that performed only ET.

ET in healthy and diabetic rats increased ERR $\alpha$ expression. ERR $\alpha$ inhibition by XCT790 did not show any significant effects on ERR $\alpha$ expression in healthy and diabetic groups. A combination of ER with ERR $\alpha$ inhibition did not change ERR $\alpha$ expression in healthy rats but on the contrary, caused a significant up-regulation of ERR $\alpha$ expression in the diabetic group which confirmed that some beneficial effects of ET are carried out thorough ERR $\alpha$.

PPAR $\alpha$ is the main isoform in the liver and involved in lipid and oxidative metabolism [10]. PPAR $\alpha$ also affects proteins that play an important role in FA transport such as CD36 and CPT1 (carnitine palmitoyltransferase 1). CPT1 involved in the process of FA transport into the mitochondria [14]. ET significantly upregulated PPAR $\alpha$ gene expression compared to control groups (healthy and diabetic controls). Tunstall et al. did not find a significant change of PPAR $\alpha$ expression after ET, which is in opposition to our data, but their study duration was only 9 days [25]. On the other hand, Horowitz and colleagues and Russel et al. reported that ET significantly increased PPAR $\alpha$ expression that in along with our findings $[21,26]$. Hence, ET by up-regulation of PPAR $\alpha$, improves FA transport and oxidation in mitochondria and decreases NEFA and, therefore, improve insulin resistance in diabetes.

PPAR $\alpha$ expression did not change significantly after ERR $\alpha$ inhibition compared to control counterparts (healthy and diabetic groups). On the other hand, a combination of ET with ERR $\alpha$ inhibition significantly increased PPAR $\alpha$ expression in healthy and diabetic rats. Our results are showing that ET effects are dominant over ERR $\alpha$ inhibition effects on PPAR $\alpha$ gene expression in ET/ERR group. We also observed the same pattern of expression by PDK4 gene and protein expression, and therefore, it seems that ET by up-regulation of PPAR $\alpha$, increases PDK4 expression. It has been proved that ET and ERR $\alpha$ regulate PPAR $\alpha$ and increase its expression [24]. Wu and coworkers have shown that PPAR $\alpha$ activation results in PDK4 gene and protein up-regulation [27] that is in accordance with our findings. It has been shown that ERR $\alpha$ up-regulation increased PPAR $\alpha$ expression [24]. Therefore, ERR $\alpha$ suppression alone reduces PPAR $\alpha$ expression, which results in PDK4 down-regulation. But, the combination of ET and ERR $\alpha$ inhibition dramatically increases PPAR $\alpha$ expression, which seems to affect PDK4 gene and protein expression in ET/ERR group. Hence, in tissue-like liver in which PPAR $\alpha$ and ERR $\alpha$ both are highly expressed, the ERR $\alpha$ dependent activation of PPAR $\alpha$ can act as a pivotal mechanism to modulate genes involved in energy metabolism [24]. 


\section{Conclusions}

Exercise training programs provide a promising alternative to improve metabolic conditions such as diabetes, atherosclerosis, and CVD. Exercise potential beneficial effects conducted thorough regulation of molecular response in target tissues such as the liver and skeletal muscle. Additionally, interventions such as ERR $\alpha$ modulations that have an important role in regulation of liver homeostasis considered as an alternative for metabolic disease management including diabetes. ERR $\alpha$ inhibits gluconeogenesis in the liver and improves metabolic oxidation in the muscle that showing two-sided effects. We showed that ERR $\alpha$ inhibition with ET has important beneficial effects compared to alone ERR $\alpha$ inhibition. Therefore, this combination needs more focus and examination to clarify its beneficial effects and further possible mechanisms in diabetic models.

\section{Funding}

This research received no external funding.

\section{Acknowledgments}

\section{This study was financially supported by the Kerman Medical University Research Council.}

\section{Conflicts of Interest}

The authors declare no conflict of interest.

\section{References}

1. Wall, C.E.; Yu, R.T.; Atkins, A.R.; Downes, M.; Evans R.M. Nuclear receptors and AMPK: can exercise mimetics cure diabetes? J Mol Endocrinol 2016, 57, R49-58, https://doi.org/10.1530/JME-16-0073.

2. Shimomura, I.; Bashmakov, Y.; Horton, J.D. Increased Levels of Nuclear SREBP-1c Associated with Fatty Livers in Two Mouse Models of Diabetes Mellitus. J. Biol. Chem 1999, 274, 30028-30032, https://doi.org/10.1074/jbc.274.42.30028.

3. Thent, Z.C.; Das, S.; Henry, L.J. Role of Exercise in the Management of Diabetes Mellitus: the Global Scenario. PLoS ONE 2013, 8, e80436, https://doi.org/10.1371/journal.pone.0080436.

4. Wende, A.R.; Huss, J.M.; Schaeffer, P.J.; Giguere, V.; Kelly, D.P. PGC-1a Coactivates PDK4 Gene Expression via the Orphan Nuclear Receptor ERRa: a Mechanism for Transcriptional Control of Muscle Glucose Metabolism. Mol Cell Biol 2005, 25, 10684-10694, https://doi.org/10.1128/MCB.25.24.1068410694.2005.

5. Abreu, P.; Vitzel, K.F.; Monteiro, I.C.C.R.; Lima, T.I.; Queiroz, A.N.; Leal-Cardoso, J.H.; Hirabar, S.M.; Ceccatto, V.M. Effects of endurance training on reduction of plasma glucose during high intensity constant and incremental speed tests in Wistar rats. Braz J Med Biol Res 2016, 49, e5226, http://doi.org/10.1590/1414431X20165226.

6. Zhang, S.; Hulver, M.W.; McMillan, R.P.; Cline, M.A.; Gilbert, E.R. The pivotal role of pyruvate dehydrogenase kinases in metabolic flexibility. Nutrition \& Metabolism 2014, 11, http://doi.org/10.1186/1743-7075-11-10.

7. Hevener, A.L.; Zhou, Z.; Moore, T.M.; Drew, B.G.; Ribas, V. The impact of ER $\alpha$ action on muscle metabolism and insulin sensitivity e Strong enough for a man, made for a woman. Mol. Metab 2018, 15, 20e34, http://doi.org/10.1016/j.molmet.2018.06.013.

8. Wada, T.; Tsuneki, H.; Sasaoka, T. New Insights Into Metabolic Regulation via Bifurcated Function of Estrogen Receptor $\alpha$. Diabetes 2013, 62, 3996-3998, http://doi.org/10.2337/db13-1325.

9. Chamkasem, A.; Toniti, W. Sequence to Structure Approach of Estrogen Receptor Alpha and Ligand Interactions. Asian Pac J Cancer Prev 2015, 16, 2161-2166, http://doi.org/10.7314/APJCP.2015.16.6.2161.

10. Fan, W.; Evans, R. PPARs and ERRs: molecular mediators of mitochondrial metabolism. Curr Opin Cell Biol 2015, 33, 49-54, http://doi.org/10.1016/j.ceb.2014.11.002.

11. Eskiocak, B.; Ali, A.; White, M.A. The Estrogen-Related Receptor $\alpha$ Inverse Agonist XCT 790 Is a nanomolar mitochondrial uncoupler. Biochemistry 2014, 53, 4839-4846, http://doi.org/10.1021/bi500737n. 
12. Huss, J.M.; Garbacz, W.G.; Xie, W. Constitutive activities of estrogen-related receptors: Transcriptional regulation of metabolism by the ERR pathways in health and disease. BBA-MOL BASIS DIS 2015, 1852, 19121927, http://doi.org/10.1016/j.bbadis.2015.06.016.

13. Audet-Walsh, E.; Giguere, V. The multiple universes of estrogen-related receptor $\alpha$ and $\gamma$ in metabolic control and related diseases. Acta Pharmacologica Sinica 2014, 121, 1-11, http://doi.org/10.1038/aps.2014.121.

14. Burri, L.; Thoresen, H.; Berge, R.K. The Role of PPAR $\alpha$ Activation in Liver and Muscle. PPAR Research 2010, 2010, 11, http://doi.org/10.1155/2010/542359.

15. Tobin, K.A.R.; Ulven, S.M.; Schuster, G.U.; Steineger, H.H.; Andresen, S.M.; Gustafsson, J.A.; Nebb, H.I. Liver X Receptors as Insulin-mediating Factors in Fatty Acid and Cholesterol Biosynthesis. J Biol Chem 2002, 277, 10691-10697, http://doi.org/10.1074/jbc.M109771200.

16. Moslehi, A.; Hamidi-zad, Z. Role of SREBPs in liver diseases: A mini-review. J Clin Transl Hepatol 2018, 6, 332-338, http://doi.org/10.14218/JCTH.2017.00061.

17. Defour, A.; Dessalle, K.; Castro Perez, A.; Poyot, T.; Castells, J.; Gallot, Y.S.; Durand, C.; Euthine, V.; Gu, Y.; Béchet, D.; Peinnequin, A.; Lefai, E.; Freyssenet, D. Sirtuin 1 Regulates SREBP-1c Expression in a LXRDependent Manner in Skeletal Muscle. PLoS ONE 2012, 7, e43490, http://doi.org/10.1371/journal.pone.0043490.

18. Steffensen, K.R.; Gustafsson, J.A. Section II: Nuclear Receptors and Islet Function Putative Metabolic Effects of the Liver X Receptor (LXR). Diabetes 2004, 53(Suppl. 1), S36-S42, https://doi.org/10.2337/diabetes.53.2007.S36.

19. Roden, M. Exercise in type 2 diabetes: to resist or to endure? Diabetologia 2012, 55, 1235-1239, https://doi.org/10.1007/s00125-012-2513-5.

20. Rezaei, S.; Molanouri Shamsi, M.; Mahdavi, M.; Jamali, A.; Prestes, J.; Tibana, R.A.; Navalta, J.W.; Voltarelli, F.A. Endurance exercise training decreased serum levels of surfactant protein D and improved aerobic fitness of obese women with type-2 diabetes. Diabetol Metab Syndr 2017, 9, https://doi.org/10.1186/s13098-017-0273-6.

21. Russell, A.P.; Feilchenfeldt, J.; Schreiber, S.; Praz, M.; Crettenand, A.; Gobelet, C.; Meier, C.A.; Bell, D.R.; Kralli, A.; Giacobino, JP.; Deriaz, O. Endurance Training in Humans Leads to Fiber Type-Specific Increases in Levels of Peroxisome Proliferator-Activated Receptor-g Coactivator-1 and Peroxisome Proliferator-Activated Receptor-g in Skeletal Muscle. Diabetes 2003, 52, 2874-2881, https://doi.org/10.2337/diabetes.52.12.2874.

22. Cauza, E.; Hanusch-Enserer, U.; Strasser, B.; Ludvik, B.; Metz-Schimmerl, S.; Pacini, G.; Wagner, O.; Georg, P.; Prager, R.; Kostner, K.; Dunky, A.; Haber, P. The Relative Benefits of Endurance and Strength Training on the Metabolic Factors and Muscle Function of People With Type 2 Diabetes Mellitus. Arch Phys Med Rehabil 2005, 86, 1527-1534, https://doi.org/10.1016/j.apmr.2005.01.007.

23. Skovgaard, C.; Brandt, N.; Pilegaard, H.; Bangsbo, J. Combined speed endurance and endurance exercise amplify the exercise-induced PGC-1a and PDK4 mRNA response in trained human muscle. Physiol Rep 2016, 4, e12864, https://doi.org/10.14814/phy2.12864.

24. Huss, J.M.; Torra, I.P.; Staels, B.; Giguere, V.; Kelly, D.P. Estrogen-Related Receptor $\alpha$ Directs Peroxisome Proliferator-Activated Receptor $\alpha$ Signaling in the Transcriptional Control of Energy Metabolism in Cardiac and Skeletal Muscle. Molecular and Cellular Biology 2004, 24, 9079-9091, https://doi.org/10.1128/MCB.24.20.9079-9091.2004.

25. Tunstall, R.J.; Mehan, K.A.; Wadley, G.D.; Collier, G.R.; Bonen, A.; Hargreaves, M.; Cameron-Smith, D. Exercise training increases lipid metabolism gene expression in human skeletal muscle. Am J Physiol Endocrinol Metab 2002, 283, E66-E72, https://doi.org/10.1152/ajpendo.00475.2001.

26. Horowitz, J.F.; Leone, T.C.; Feng, W.; Kelly, D.P.; Klein, S. Effect of endurance training on lipid metabolism in women: a potential role for PPAR alpha in the metabolic response to training. Am J Physiol Endocrinol Metab 2000, 279, E348-E355, https://doi.org/10.1152/ajpendo.2000.279.2.E348.

27. Wu, P.; Inskeep, K.; Bowker-Kinley, M.M.; Popov, K.M.; Harris, R.A. Mechanism responsible for inactivation of muscle pyruvate dehydrogenase complex in starvation and diabetes. Diabetes 1999, 48, 15931599, https://doi.org/10.2337/diabetes.48.8.1593.

28. Thomas, A.W.; Davies, N.A.; Moir, H.; Watkeys, L.; Ruffino, J.S.; Isa, S.A. Exercise-associated generation

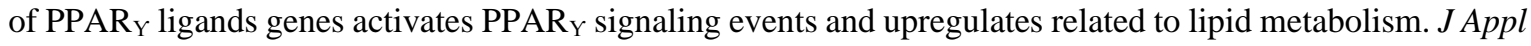
Physiol 2012, 112, 806-815, https://doi.org/10.1152/japplphysiol.00864.2011.

29. Kazeminasab, F.; Marandi, M.; Ghaedi, K.; Esfarjani, F.; Moshtaghian, J. Endurance training enhances LXRa gene expression in Wistar male rats. Eur $J$ Appl Physiol 2013, 113, 2285-2290, https://doi.org/10.1007/s00421-013-2658-z.

30. Jeoung, N.H.; Harris, R.A. Role of Pyruvate Dehydrogenase Kinase 4 in Regulation of Blood Glucose Levels. Korean diabetes J. 2010, 34, 274-283, https://doi.org/10.4093/kdj.2010.34.5.274.

31. Hu, J.Z.; Long, H.; Wu, T.D.; Zhou, Y.; Lu, H.B. The effect of Estrogen-related receptor $\alpha$ on the regulation of angiogenesis after spinal cord injury. Neuroscience 2015, 290, 570-580, https://doi.org/10.1016/j.neuroscience.2015.01.067. 
32. Aminizadeh, S.; Masoumi-Ardakani, Y.; Shahouzehi, B. The effects of PDK4 inhibition on AMPK protein levels and PGC-1 $\alpha$ gene expression following endurance training in skeletal muscle of Wistar rats. $U k r$. Biochem. J 2018, 90, 89-96, https://doi.org/10.15407/ubj90.06.089.

33. Mohammadi, A.; Fallah, H.; Shahouzehi, B.; Najafipour, H. miR-33 inhibition attenuates the effect of liver X receptor agonist T0901317 on expression of liver X receptor alpha in mice liver. ARYA Atheroscler 2017, 13, 257-63.

34. Sigal, R.J.; Kenny, G.P.; Wasserman, D.H.; Castaneda-Sceppa, C. Physical Activity/Exercise and Type 2 Diabetes. Diabetes Care 2004, 27, 2518-2539, https://doi.org/10.2337/diacare.27.10.2518.

35. Baghersalimi, M. Effect of Exercise on Carbohydrate and Lipid Metabolism in the Liver. Int. J App. Sci. Physic. Edu 2018, 2, 42-57.

36. Jeong, J.Y.; Jeoung, N.H.; Park, K.G.; Lee, I.K. Transcriptional Regulation of Pyruvate Dehydrogenase Kinase. Diabetes Metab J 2012, 36, 328-335, https://doi.org/10.4093/dmj.2012.36.5.328. 